\title{
Volume Is Not the Only Key to Hypertension Control in Dialysis Patients
}

\author{
Aghogho Odudu $^{a, b}$ Christopher Mclntyre ${ }^{a, b}$ \\ ${ }^{a}$ Department of Renal Medicine, Derby Hospitals NHS Foundation Trust, Derby, and ${ }^{b}$ Graduate Entry Medical \\ School, University of Nottingham, Nottingham, UK
}

\section{Key Words}

Haemodialysis - Hypertension • Volume expansion •

Peritoneal dialysis $\cdot$ Sodium

\begin{abstract}
There is a widely held belief that hypervolaemia due to excess intake or inadequate removal of salt and water is the principal cause of hypertension in dialysis patients. The risk of failing to consider additional pathophysiological elements is that inadequate or inappropriate therapeutic strategies may be adopted. This review aims to highlight multiple alternative mechanisms for hypertension in this setting along with the risks of probing for normotension by empirical dry weight reduction if dry weight is imprecisely defined.
\end{abstract}

Copyright $\odot 2012$ S. Karger AG, Basel

\section{Introduction - Hydration Status and Empirical Association with Blood Pressure}

There is a high prevalence of hypertension in endstage renal disease (ESRD) with estimates from 25 to $80 \%$. The cause of this has been principally attributed to loss of renal regulation of salt and water excretion leading to extracellular volume overload with consequent adverse cardiac remodelling $[1,2]$. As such, the principle approach is to use a combination of ultrafiltration by dialysis to dry weight and antihypertensive therapy to control blood pressure (BP). There is a strong and linear risk association of BP to cardiovascular morbidity in the general population with the risk reduced by drug therapy. In ESRD, the relationship of risk with hypertension is fundamentally more complicated. Large observational studies of haemodialysis (HD) patients consistently show a J- or U-shaped association, with a paradoxical survival advantage in hypertensive patients and the highest mortality in hypotensive patients after multivariable adjustment [3]. Based on these data some have cautioned against using drug therapy to lower BP in hypertensive dialysis patients. Detailed discussion of this controversy is beyond the scope of this review. However, meta-analyses suggested benefit of drug therapy, but the heterogeneity of the trials did not allow the benefit to be isolated to a particular class nor could the interaction with volume status be examined [4].

The existence of overhydration in hypertensive dialysis patients is far from universal. Wabel et al., measured pre-dialysis systolic BP and fluid status by bioimpedance in $500 \mathrm{HD}$ patients compared to a matched healthy population [5]. Only $15 \%$ of patients fitted the stereotype of overhydration with hypertension. $13 \%$ of patients had hypertension despite underhydration and 10\% had overhydration despite normal or low BP. Similar findings were seen in another study using the same methodology in 639 patients using peritoneal dialysis (PD) [6]. These studies highlight that although physicians often estimate hydration status by BP, the two factors are often dissociated in the setting of the physiological derangements characteristic of ESRD. A wide variety of pathophysiological mechanisms may contribute to this dissociation.

\section{KARGER}

Fax +4161306 1234

E-Mail karger@karger.ch

www.karger.com
(C) 2012 S. Karger AG, Basel

$1660-2110 / 12 / 1203-0173 \$ 38.00 / 0$

Accessible online at:

www.karger.com/nec
Christopher McIntyre

Department of Renal Medicine, Derby Hospital NHS Foundation Trust Uttoxeter Road

Derby DE22 3NE (UK)

Tel. +44 133278 9344, E-Mail aghogho.odudu@nhs.net 


\section{Overactivity of the Renin-Angiotensin System}

Despite losing renal excretory function, dialysis patients have inappropriately high renin levels relative to their salt and volume status [7]. A subgroup of patients with volume-independent hypertension do not respond to salt or volume removal but are sensitive to ACE inhibitors [7]. This subgroup can have hypertension despite low volume.

\section{Increased Arterial Stiffness}

Arterial stiffness resulting from calcifying or non-calcifying alterations in the arterial wall is a characteristic consequence of advanced kidney disease. Blacher et al. [8] performed a study of 241 patients, divided into 3 groups depending on the degree of central arterial stiffness determined by pulse wave velocity. After 6 years of followup, those with the greatest stiffness had the highest mortality and this was more prognostic than age or BP. Our group assessed the relationship of vascular calcification to arterial stiffness and its progression with time in 134 patients (60 on HD, 28 on PD, 46 with stage $4 \mathrm{CKD}$ ) assessed at baseline and 2 years by femoral artery computed tomography and pulse wave velocity. We showed increased arterial stiffness correlated with vascular calcification and increased with progressive stage of CKD. Vascular calcification was strongly predictive of death, being present in 20 of the 21 patients who died. This was highly correlated with decreasing diastolic BP and increasing pulse pressure [9]. In this setting, the principal driver of hypertension is not overhydration but a reduction in arterial compliance. This allows hypertension to occur despite normal hydration or underhydration. Arterial stiffness will also further aggravate hypertension in those with overhydration.

\section{Advanced Glycation End Product Tissue Deposition}

A relatively novel risk factor in increasing arterial stiffness is the formation and tissue deposition of advanced glycation end products (AGEs). These heterogeneous compounds were classically described as end products of a complex non-enzymatic reaction between glucose and proteins with a long half-life such as collagen. It is now known that AGEs accumulate by a variety of nonglucose-mediated methods including ingested processed food, hyperlipidaemia, oxidative stress, carbonyl stress and reduced renal clearance. AGE formation has therefore been proposed to be a direct result of cumulative metabolic stress [10]. AGE accumulation causes crosslinking of collagen fibres directly increasing arterial stiffness. AGE levels are markedly increased in dialysis patients and predictive of mortality [10]. This may be as a result of reduced arterial compliance and increased endothelial dysfunction.

\section{The Lag Phenomenon and Buffering of Water-Free Sodium}

The Tassin group coined the term 'lag phenomenon' to explain a well-described time delay between normalization of extracellular volume in HD patients by ultrafiltration and a sustained further reduction in BP. This lag lasted several months, highlighting a dissociation between hypertension and hydration state [11]. The explanation for this may be linked to Jens Titze's work showing that large amounts of sodium can be buffered in the interstitial space without commensurate water retention [12]. Accumulation within resistance vessels may also contribute to increased tonic vasoconstriction, further favouring the development of hypertension. This extension to the traditional model of fluid homeostasis is supported by data showing that sodium is proinflammatory and is capable of direct adverse cardiovascular consequences independent of its effect on BP $[12,13]$.

\section{The Role of Hyperglycaemia in Determining Extracellular Volume}

Hyperglycaemia may be another factor in the dissociation of BP and hydration status. Patients with diabetes ingest water as a result of glucose-driven hypertonicity. Ramdeen et al. [14] performed a one-year balance study in 9 diabetic and 7 non-diabetic HD patients. This demonstrated sodium intake with commensurate water retention accounted for the entire inter-dialytic fluid gains in non-diabetic patients but only half the fluid gains in diabetic patients. The other half was pure water intake presumably driven by hyperglycaemia. In this setting, free water ingestion is not being driven by the conventional salt and water paradigm, and overhydration may even occur without hypertension.

Acute hyperglycaemia and hyperinsulinaemia can directly cause hypertension via vasopressor effects [15]. We have previously demonstrated that PD exchanges with 
hypertonic glucose-based dialysate causes sustained hyperglycaemia and hyperinsulinaemia with resultant hypertension in both the fed and starved state [16]. In this setting, hypertension can occur despite the dehydrating effect of peritoneal ultrafiltration and is abrogated by the use of glucose-sparing dialysate.

\section{Perils of Probing for Normotension by Empirical Dry Weight Reduction}

There are inherent difficulties in using volume control to achieve normal BP and dry weight. Firstly, the component or timing of BP to target is not clear. Secondly, dry weight is empirically and imprecisely defined. Overhydration should be assiduously avoided, but we must also aim to achieve dry weight without causing underhydration and dialysis-related haemodynamic instability. Agarwal [17] studied $326 \mathrm{HD}$ patients for a mean of 32 months and showed that home-monitored systolic BP was strongly associated with mortality, whilst diastolic $\mathrm{BP}$ and $\mathrm{BP}$ taken on the dialysis unit had no prognostic value. There was a bimodal distribution with the lowest mortality at systolic BP $110-130 \mathrm{~mm} \mathrm{Hg}$ and highest mortality in both hypotensive and hypertensive patients. Furthermore, a significant portion of dialysis patients have attenuated diurnal variation in $\mathrm{BP}$ and it is speculated that this may mediate enhanced cardiovascular risk [18]. These data are concordant with Agarwal's finding that home-measured and ambulatory $\mathrm{BP}$ is the most useful guide to treatment.

\section{Systemic Circulatory Stress and Cardiac Injury}

Dialysis patients are uniquely susceptible to demand myocardial ischaemia. Their greater arterial stiffness means systolic pulse waves are more likely to be reflected from the periphery during diastole, shortening coronary filling time. In this way, arterial stiffness lowers the ischaemic threshold allowing myocardial ischaemia to occur even in the absence of large vessel coronary artery disease. This reduced coronary flow reserve is well described in dialysis patients [19]. To that end, it is unsurprising that lower diastolic BP is associated with greater vascular calcification, arterial stiffness and mortality in this setting $[8,9]$. Dialysis patients also have endothelial dysfunction and impaired autoregulation of BP leaving the heart less able to buffer volume changes during HD. We performed a study demonstrating HD patients with the least barore-

Volume Control and Blood Pressure in Dialysis Patients flex sensitivity were more likely to have intradialytic hypotension [20]. Our proof-of-concept study in HD patients who had recent normal coronary angiography demonstrated that HD was characterised by a $30 \%$ global reduction in myocardial blood flow using the gold standard of $\mathrm{H}_{2}{ }^{15} \mathrm{O}$ positron emission tomography [21]. The ischaemic segments matched segmental regional wall motion abnormalities (RWMAs) identified by simultaneous 2-D echocardiography at peak stress (225 min into an HD session) compared to before HD [21]. This supported the use of stress RWMAs by echocardiography as a surrogate measure of myocardial ischaemia as it was a feasible imaging modality for larger numbers of patients. Our subsequent natural history study in 70 prevalent HD patients showed that compared to pre-HD echocardiography, new RWMAs developed at peak stress in 45/70 participants, and this associated with $20 \%$ mortality and $13 \%$ reduction in mean left ventricular ejection fraction after 1 year [22]. All segmental RWMAs in the 36/45 survivors persisted at follow-up. In the cohort who had no abnormal segments at the start of the study, new segmental RWMAs had developed. Multivariable regression analysis identified significant determinants of these stress RWMAs as age, ultrafiltration volume, severity of intradialytic hypotension and serum troponin T concentration, displacing traditional risk variables including diabetes status and history of ischaemic heart disease [22]. In short, the RWMAs characterise those who suffer ischaemia due to the haemodynamic stress of HD, and this might be limited by alternative strategies such as longer or more frequent HD.

\section{Potential Risks of Overshooting Dry Weight in Other Vascular Beds}

Probing for dry weight may also be at the risk of underperfusing other vascular beds including those of the kidney, gut and brain. Although it might seem counterintuitive to be concerned about renal perfusion in a dialysis patient, it is well recognised that maintenance of residual renal function confers a survival advantage and improves volume control. Günal et al. [23] targeted normal BP as a surrogate for normal volume status in $78 \mathrm{PD}$ patients by increasing ultrafiltration volumes with $3.86 \%$ glucose dialysate and salt restriction. They showed an impressive reduction in $\mathrm{BP}$ to a normotensive range at the expense of a $28 \%$ decrease in urine volume in the 19 patients with significant residual renal function. The gut is also an important vascular bed, extracting $25 \%$ of resting 
cardiac output to maintain nutrition. $70 \%$ of gut bacteria possess profoundly proinflammatory and cardiotoxic endotoxin. In health, protection from endotoxin is afforded by energy-dependent tight junctions in the gut wall. Endotoxin translocation across leaking tight junctions in disease states with aberrant perfusion such as advanced heart failure and septic shock is well described in the study by Charalambous et al. [24]. We hypothesised that dialysis might also lead to aberrant gut perfusion and endotoxaemia and performed a study in 249 subjects without heart failure across the spectrum of CKD. Serum endotoxin levels were 1,000 times greater in HD patients compared to non-CKD patients, with a fourfold increase between pre-dialysis CKD stage 5 and commencing HD or PD [25]. These elevated levels correlated with intradialytic hypotension, systemic inflammation, and troponin T levels. Whether dialysis-associated endotoxaemia is a culprit or bystander in the pathophysiology of inflammation, malnutrition and adverse cardiovascular outcomes in this setting is subject to current studies. We have further proposed and are investigating the hypothesis that the cerebral microcirculation is also sensitive to dialysis-induced circulatory stress potentially explaining specific patterns of brain injury highly prevalent in the dialysis setting [26].

\section{Optimising Hydration State without Jeopardy to Critical Vascular Beds}

The frequent risk of dialysis-related hypoperfusion is due to imprecision in the assessment of dry weight. If dry weight could be accurately, reliably and practicably determined, then we would not have to resort to probing for this by trial and error with the risks we have outlined. A detailed review of practical technologies that assist in optimising hydration is beyond the scope of this review, but perhaps the most promising is bioimpedance analysis. This measures resistance which is proportional to tissue water and reactance which is proportional to cell mass, providing an index of body composition which can be longitudinally monitored. The difficulty arises in converting this measure of relative hydration into absolute volumes of fluid as this requires algorithms using assumptions from studies of healthy volunteers that perform poorly when applied to populations with disordered hydration. We conducted a study showing the added value of combining the relative hydration data from bioimpedance with the absolute hydration data using a novel deuterium breath-test by portable mass spectrometer.
This highlighted that higher co-morbidity scores led to increasing underestimation of extracellular volume by bioimpedance [27]. Recently, two novel approaches to quantify excess hydration by bioimpedance have been proposed. Assessment of continuous intradialytic calf bioimpedance ratios does not rely on geometric assumptions or comparison to population averages typical of whole-body methods [24]. Chamney et al. [28] proposed a three-compartment physiological tissue model allowing quantification of excess fluid compared to normal controls. Whilst requiring a population reference standard, the unique separation of extracellular volume into that due to excess and normal hydration is an important conceptual advance and has been shown to be of prognostic value in predicting mortality in a HD population [29]. Machek et al. [30] used this new approach to perform bioimpedance-guided prescription of dry weight in 52 HD patients. They showed that not only did this assist in dealing with hypertension in the overhydrated, but it enabled a $73 \%$ reduction in intradialytic adverse events by increasing the target weight in the underhydrated group. These preliminary data are promising, but whether this will be of a discernible long-term value requires studies randomising clinicians to be blinded or have access to bioimpedance data, and such studies have commenced (ISRCTN 95439739).

\section{Conclusion}

In summary, overhydration does not always equate to hypertension. Factors such as arterial stiffness, high renin states, the osmotic effects of glucose and the nonosmotic effects of sodium lead to a complex interplay. We have delineated some of the risks inherent in using a BPdominated definition of dry weight. Bioimpedance techniques show promise in assisting with a precise determination of hydration status allowing clinicians to control volume and $\mathrm{BP}$ without jeopardising short- or long-term perfusion to critical vascular beds.

\section{Disclosure Statement}

The authors declare no competing interests. Dr. Odudu is funded by a British Heart Foundation Clinical Research Training Fellowship Grant. 


\section{References}

1 Foley RN, Parfrey PS, Harnett JD, Kent GM, Murray DC, Barre PE: Impact of hypertension on cardiomyopathy, morbidity and mortality in end-stage renal disease. Kidney Int 1996;49:1379-1385.

-2 Kalantar-Zadeh K, Regidor DL, Kovesdy CP, Van Wyck D, Bunnapradist S, Horwich TB, Fonarow GC: Fluid retention is associated with cardiovascular mortality in patients undergoing long-term hemodialysis. Circulation 2009;119:671-679.

3 Kalantar-Zadeh K, Kilpatrick RD, McAllister CJ, Greenland S, Kopple JD: Reverse epidemiology of hypertension and cardiovascular death in the hemodialysis population. Hypertension 2005;45:811-817.

4 Agarwal R, Sinha AD: Cardiovascular protection with antihypertensive drugs in dialysis patients. Hypertension 2009;53:860-866.

$\checkmark 5$ Wabel P, Moissl U, Chamney P, Jirka T, Machek P, Ponce P, Taborsky P, Tetta C, Velasco N, Vlasak J, Zaluska W, Wizemann V: Towards improved cardiovascular management: the necessity of combining blood pressure and fluid overload. Nephrol Dial Transplant 2008;23:2965-2971.

-6 Van Biesen W, Williams JD, Covic AC, Fan S, Claes K, Lichodziejewska-Niemierko $M$, Verger C, Steiger J, Schoder V, Wabel P, Gauly A, Himmele R, Euro BCMSG: Fluid status in peritoneal dialysis patients: The European Body Composition Monitoring (EuroBCM) study cohort. PloS One 2011;6:e17148.

7 Doulton TW, MacGregor GA: Review: blood pressure in haemodialysis patients: the importance of the relationship between the renin-angiotensin-aldosterone system, salt intake and extracellular volume. J Renin Angiotensin Aldosterone Syst 2004;5:14-22.

-8 Blacher J, Guerin AP, Pannier B, Marchais SJ, Safar ME, London GM: Impact of aortic stiffness on survival in end-stage renal disease. Circulation 1999;99:2434-2439.

9 Sigrist MK, Taal MW, Bungay P, McIntyre CW: Progressive vascular calcification over 2 years is associated with arterial stiffening and increased mortality in patients with stages 4 and 5 chronic kidney disease. Clin J Am Soc Nephrol 2007;2:1241-1248.
10 Meerwaldt R, Hartog JWL, Graaff R, Huisman RJ, Links TP, den Hollander NC, Thorpe SR, Baynes JW, Navis G, Gans ROB, Smit AJ: Skin autofluorescence, a measure of cumulative metabolic stress and advanced glycation end products, predicts mortality in hemodialysis patients. J Am Soc Nephrol 2005;16:3687-3693.

11 Charra B, Bergstrom J, Scribner BH: Blood pressure control in dialysis patients: importance of the lag phenomenon. Am J Kidney Dis 1998;32:720-724.

12 Titze J: Water-free $\mathrm{Na}^{+}$retention: interaction with hypertension and tissue hydration. Blood Purif 2008;26:95-99.

13 Oberleithner H, Kusche-Vihrog K, Schillers $\mathrm{H}$ : Endothelial cells as vascular salt sensors. Kidney Int 2010;77:490-494.

14 Ramdeen G, Tzamaloukas AH, Malhotra D, Leger A, Murata GH: Estimates of interdialytic sodium and water intake based on the balance principle: differences between nondiabetic and diabetic subjects on hemodialysis. ASAIO J 1998;44:812-817.

15 Muniyappa R, Montagnani M, Koh KK, Quon MJ: Cardiovascular actions of insulin. Endocr Rev 2007;28:463-491.

16 Selby NM, Fialova J, Burton JO, McIntyre CW: The haemodynamic and metabolic effects of hypertonic-glucose and amino-acidbased peritoneal dialysis fluids. Nephrol Dial Transplant 2007;22:870-879.

17 Agarwal R: Blood pressure and mortality among hemodialysis patients. Hypertension 2010;55:762-768.

18 Jafar TH, Ebrahim SB: Variations in levels of blood pressure: of prognostic value or not? Am J Kidney Dis 2008;52:638-641.

19 Niizuma S, Takiuchi S, Okada S, Horio T, Kamide K, Nakata H, Yoshihara F, Nakamura S, Kawano Y, Nakahama H, Iwanaga Y, Nakatani S: Decreased coronary flow reserve in haemodialysis patients. Nephrol Dial Transplant 2008;23:2324-2328.

20 Chesterton LJ, Selby NM, Burton JO, Fialova J, Chan C, McIntyre CW: Categorization of the hemodynamic response to hemodialysis: the importance of baroreflex sensitivity. Hemodial Int 2010;14:18-28.
21 McIntyre CW, Burton JO, Selby NM, Leccisotti L, Korsheed S, Baker CSR, Camici PG: Hemodialysis-induced cardiac dysfunction is associated with an acute reduction in global and segmental myocardial blood flow. Clin J Am Soc Nephrol 2008;3:19-26.

22 Burton JO, Jefferies HJ, Selby NM, McIntyre CW: Hemodialysis-induced cardiac injury: determinants and associated outcomes. Clin J Am Soc Nephrol 2009;4:914-920.

-23 Günal AI, Duman S, Özkahya M, Töz H, Asçi G, Akçiçek F, Basçi A: Strict volume control normalizes hypertension in peritoneal dialysis patients. Am J Kidney Dis 2001; 37:588-593.

24 Charalambous BM, Stephens RC, Feavers IM, Montgomery HE: Role of bacterial endotoxin in chronic heart failure: the gut of the matter. Shock 2007;28:15-23.

25 McIntyre CW, Harrison LE, Eldehni MT, Jefferies HJ, Szeto CC, John SG, Sigrist MK, Burton JO, Hothi D, Korsheed S, Owen PJ, Lai KB, Li PK: Circulating endotoxemia: a novel factor in systemic inflammation and cardiovascular disease in chronic kidney disease. Clin J Am Soc Nephrol 2011;6:133141.

26 Eldehni MT, McIntyre CW: Does haemodialysis result in brain injury? Semin Dial, in press.

27 Chan C, McIntyre C, Smith D, Spanel P, Davies SJ: Combining near-subject absolute and relative measures of longitudinal hydration in hemodialysis. Clin J Am Soc Nephrol 2009;4:1791-1798.

28 Chamney PW, Wabel P, Moissl UM, Muller MJ, Bosy-Westphal A, Korth O, Fuller NJ: A whole-body model to distinguish excess fluid from the hydration of major body tissues. Am J Clin Nutr 2007;85:80-89.

29 Wizemann V, Wabel P, Chamney P, Zaluska W, Moissl U, Rode C, Malecka-Masalska T, Marcelli D: The mortality risk of overhydration in haemodialysis patients. Nephrol Dial Transplant 2009;24:1574-1579.

30 Machek P, Jirka T, Moissl U, Chamney P, Wabel P: Guided optimization of fluid status in haemodialysis patients. Nephrol Dial Transplant 2010;25:538-544. 